\title{
Spectral polarimetry technique as a complementary tool to ellipsometry of dielectric films
}

\author{
Marcelo B. Pereira, ${ }^{\star}$ Bruno J. Barreto, and Flavio Horowitz \\ Instituto de Física-Universidade Federal do Rio Grande do Sul (UFRGS), \\ Campus do Vale CP15051-91501-970 Porto Alegre, RS, Brazil \\ *Corresponding author: marcelo.barbalho@ufrgs.br \\ Received 13 January 2011; accepted 13 January 2011; \\ posted 19 January 2011 (Doc. ID 141102); published 9 March 2011
}

\begin{abstract}
Ellipsometry is a highly sensitive optical technique for coating characterization but usually presents multiple solutions in many cases. To prevent these, a method with addition of a spectral polarimetric technique is proposed. An initial film dispersion curve, independently of its physical thickness, is then provided using the same setup as spectral ellipsometry and at the same sample position, which later is used for thickness determination and dispersion refinement with increase of reliability of results. Characterization of thin $\mathrm{TiO}_{2}$ films with one and two ellipsometric solutions is shown to corroborate the validity of the proposed method. (C) 2011 Optical Society of America

OCIS codes: $\quad 310.6860,120.2130$.
\end{abstract}

\section{Introduction}

Spectral ellipsometry is one of the most powerful tools used to investigate optical materials $[\underline{1}, 2]$. In the case of a simple, homogeneous dielectric thin film, the technique allows determination of its optical constants: physical thickness, $d$, and refractive index as function of light wavelength, i.e., the dispersion curve $n(\lambda)$.

As in the envelope [3] and M-Line [4] methods, ellipsometry is based on film optical thickness, the product of $n(\lambda)$ and $d$, which is determined by the changes of optical polarization caused by the interaction between the light probe beam and the sample [5]. The optical constants are related with the polarization state of light by the ellipsometry fundamental equation in terms of the Drude reflection coefficients $[\underline{1}, \underline{5}]$ :

$$
\frac{r_{p}\left(n_{0}(\lambda), n_{s}(\lambda), n(\lambda), d, \theta\right)}{r_{s}\left(n_{0}(\lambda), n_{s}(\lambda), n(\lambda), d, \theta\right)}=e^{-i \Delta} \tan (\psi),
$$

where $n_{0}(\lambda)$ and $n_{s}(\lambda)$ are, respectively, the dispersion curves of air and substrate, $\theta$ is the light incident

0003-6935/11/09C420-04\$15.00/0

(C) 2011 Optical Society of America angle, and $\Delta$ and $\tan (\Psi)$ are the phase shift and amplitude ratio, respectively, between the $p$ and $s$ polarization components of light.

Even for dielectric isotropic films, the ellipsometric solution of this equation usually is not unique. In this case, previous knowledge of the film under study is required: either an estimate of its thickness or of its dispersion curve. Another alternative is to try to decouple the film optical constants (refractive index and physical thickness) by performing measurements at multiple angles. More detailed information about spectral ellipsometry can be seen in $[1,2, \underline{2}-7]$.

In this context, we present a characterization method that uses spectral polarimetry to obtain an estimate of the film dispersion curve, independently of its physical thickness, which is later used in the analysis of spectral ellipsometry data.

\section{Theory}

Differently from the other traditional methods previously mentioned, the Abelès technique uses $p$ polarized light to measure only the refractive index of thin films [8]. In this method, light reflection intensities from the film and from the bare substrate are compared at variable incident angles $(\theta)$ (see Fig. 1). 


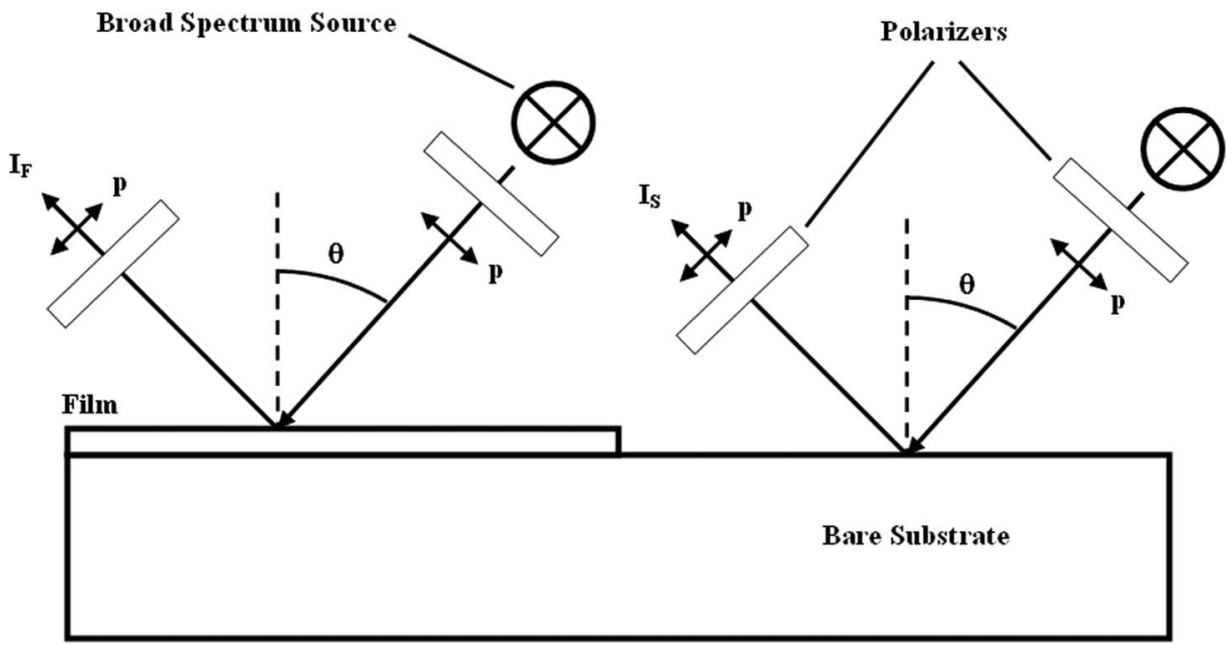

Fig. 1. Scheme used in spectral polarimetry measurements. Light from a broadband spectral source is polarized ( $p$ polarization) and strikes the film or the bare substrate at a variable incident angle $\theta$. The intensities of the light ( $p$ polarization) reflected by the coated $\left(I_{F}\right)$ and by the uncoated substrate $\left(I_{S}\right)$ are compared, and their matching angular position is located at a certain wavelength value.

The match between light reflection intensities is achieved in two particular cases: when the film optical thickness causes destructive interference in reflection among the multiple beams that travel inside the film [9] (observed for both $p$ - and $s$-polarized light) or when the incident angle corresponds to the Brewster condition at the air-film interface for a specific light wavelength [10]. In this case, the corresponding refractive index for this wavelength is obtained by the well known relation [11]

$$
n=\tan \theta_{B}
$$

where $\theta_{B}$ is the Brewster angle for a fixed light wavelength.

A limitation in Abelès approach is that only one angular position is measured at the minimum of a broad saddle in the reflectance difference curve, which lowers precision of the method. One order of magnitude is gained with Hacskaylo extension [12], which introduces other measurement data with a small deviation from $p$-polarized light.

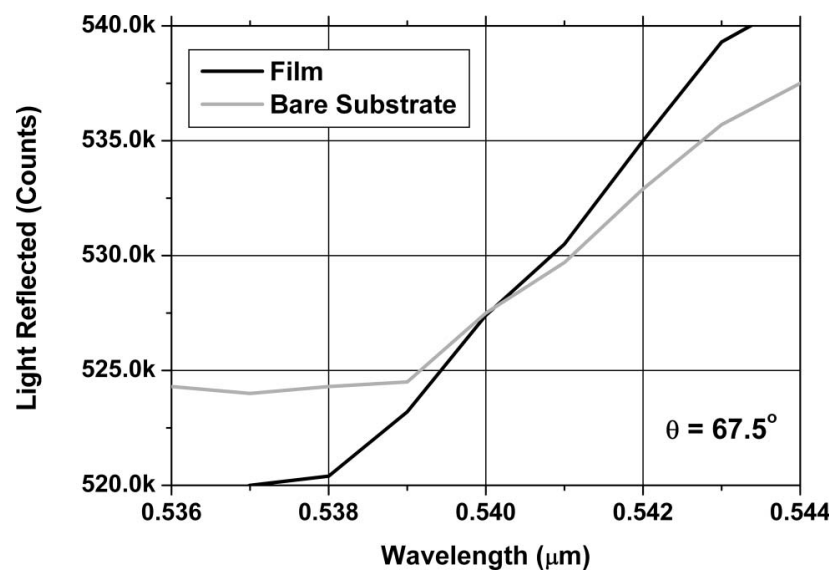

Fig. 2. Intersection between film and bare substrate reflection spectra for sample $1\left(\theta=67.5^{\circ}\right)$.
For the spectral case, a set of data is now determined at different wavelengths with dispersion considered, that is

$$
n(\lambda)=\tan \theta_{B}(\lambda)
$$

where a continuous dispersion curve is reasonably assumed, and outside from any near resonance anomalous region.

\section{Experiment}

To test the characterization method that combines spectral polarimetry and ellipsometry techniques, two dielectric thin films of $\mathrm{TiO}_{2}$ were deposited on half of the silicon substrates, using an RF-Magnetron Sputtering system from Leybold.

The first film, labeled sample 1, was prepared to be a very thin film with $42 \mathrm{~nm}$ ( $1 \mathrm{~h}$ of deposition using $250 \mathrm{~W}$ ). Since this film is very thin, only one solution is expected from spectral ellipsometry, which then can be used as proof of the method.

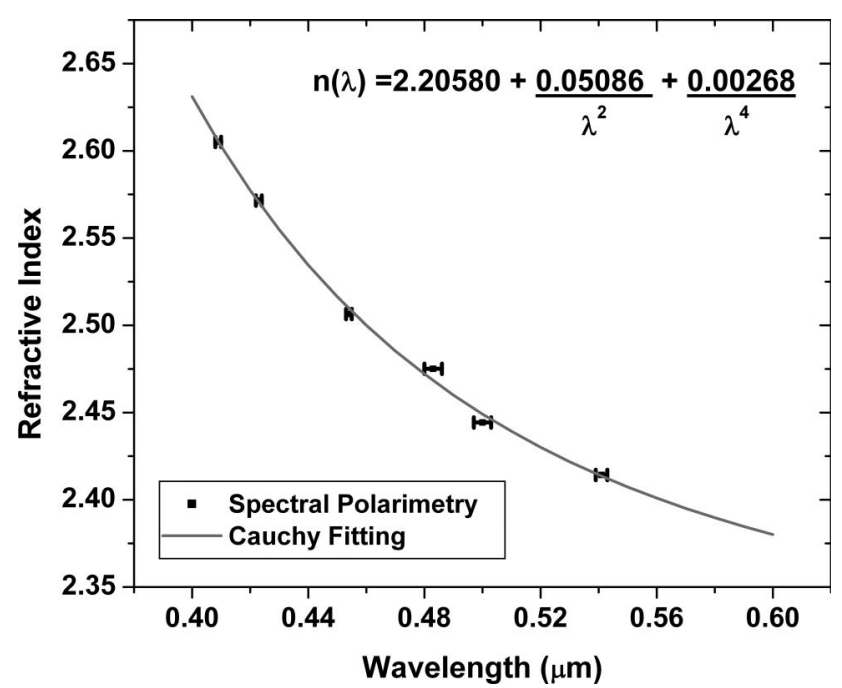

Fig. 3. Spectral polarimetry data and Cauchy curve fitting (sample 1). 
Table 2. Solutions Obtained for the Sample 2 Using Two Approaches, With and Without the Polarimetric Estimate ${ }^{a}$

\begin{tabular}{|c|c|c|c|c|}
\hline \multirow[b]{2}{*}{ Cauchy Coefficients } & \multicolumn{2}{|c|}{ Ellipsometry Only } & \multicolumn{2}{|c|}{ Polarimetry/Ellipsometry } \\
\hline & Solution 1 & Solution 2 & Polarimetry Estimate & Ellipsometry \\
\hline$A$ & $2.197 \pm 0.003$ & $2.951 \pm 0.003$ & 2.05410 & $2.198 \pm 0.003$ \\
\hline$C$ & $0.00794 \pm 0.00009$ & $0.02343 \pm 0.00009$ & 0.01623 & $0.00795 \pm 0.00009$ \\
\hline Thickness $(\mu \mathrm{m})$ & $0.1954 \pm 0.0003$ & $0.3211 \pm 0.0005$ & No & $0.1954 \pm 0.0003$ \\
\hline$R^{2}$ & 0.992 & 0.959 & No & 0.992 \\
\hline
\end{tabular}

${ }^{a}$ Ellipsometry alone provides two solutions for the $\mathrm{TiO}_{2}$ film; after the polarimetric method estimate, only the correct solution results.

The second film, labeled sample 2, was deposited for $10 \mathrm{~h}(250 \mathrm{~W})$ and, to decrease film inhomogeneities, the sample was kept in rotation $(12 \mathrm{rpm})$ and heated $\left(80^{\circ} \mathrm{C}\right)$ during all deposition processes. This sample was produced to obtain multiple solutions in spectral ellipsometry, so that the dispersion curve coefficients calculated from the polarimetric results could be used to exclude unreal solutions obtained from ellipsometry.

At first, sample 1 was measured using an ellipsometer SOPRA GES-5E (wavelength range of 0.35 to $0.85 \mu \mathrm{m}$, at $\theta=75^{\circ}$ ). Afterwards, polarizers were set to $p$ polarization and spectral polarimetry measurements were performed in the wavelength range of 0.4 to $0.6 \mu \mathrm{m}$ (step of $0.001 \mu \mathrm{m}$ ), with incident angle range of 67.5 to $69^{\circ}$ (step of $0.25^{\circ}$ ). For each incident angle, the light beam incident on first the film and reflection intensity values were collected; later the sample was dislocated by a translation stage until the beam incident on only the bare substrate, and again intensity values were collected.

Figure 2 shows the intersection between film and bare substrate reflection spectra for the measurement performed at $\theta=67.5^{\circ}$. For this particular incident angle, the intersection indicates the light wavelength at which the Brewster condition is satisfied. With the same procedure for the other measurement angles, adjustment of a Cauchy function was possible [13] (see Fig. 3), as well as determination of its coefficients: $A=2.20580, B=0.05086$, and $C=0.00268$.

These coefficient values were then used in the ellipsometer analysis-modeling software, as an initial estimate for the dispersion curve of the film. As a result, the software converges quickly to a solution that fits to the experimental data with a coefficient of de-

Table 1. Refractive Index Values for the $\mathrm{TiO}_{2}$ Film in Sample 1, Obtained from Polarimetry (Estimate, see Fig. $\underline{3}$ ) and Ellipsometry

\begin{tabular}{ccc}
\hline \multicolumn{3}{c}{ Refractive Index } \\
\hline$\lambda(\mu \mathrm{m})$ & Polarimetry $( \pm 0.03$, estimate $)$ & Ellipsometry \\
\hline 0.425 & 2.57 & $2.57 \pm 0.01$ \\
0.450 & 2.52 & $2.50 \pm 0.01$ \\
0.475 & 2.48 & $2.451 \pm 0.009$ \\
0.500 & 2.45 & $2.417 \pm 0.008$ \\
0.525 & 2.43 & $2.394 \pm 0.008$ \\
0.550 & 2.41 & $2.379 \pm 0.007$ \\
0.575 & 2.39 & $2.368 \pm 0.007$ \\
0.600 & 2.38 & $2.362 \pm 0.007$ \\
\hline
\end{tabular}

termination $\left(R^{2}\right)$ of 0.9952 [13], with film thickness $d=0.0424 \pm 0.001 \mu \mathrm{m}$ and $\overline{C a u c h y}$ coefficients $A=$ $2.448 \pm 0.005, \quad B=-0.0845 \pm 0.0006, \quad$ and $C=$ $0.01921 \pm 0.00008$. A comparison between film refractive index values, obtained by polarimetry and ellipsometry, is shown in Table 1 for some visible wavelengths.

This thinner optical $\mathrm{TiO}_{2}$ film allows a single ellipsometric solution at each wavelength, which coincides with that from the proposed method, well within the measurement uncertainties, thus corroborating its validity.

The same measurement procedure was performed with sample 2. Polarimetric measurements ranged between $66^{\circ}$ and $69^{\circ}$ in incident angle (step of $0.25^{\circ}$ ), and $0.4-0.65 \mu \mathrm{m}$ in wavelength (step of $0.001 \mu \mathrm{m}$ ). In the first approach, without use of the dispersion curve from the polarimetric method, the ellipsometer analysis-modeling software obtained two solutions with different sets of Cauchy coefficients and film thicknesses, as shown in columns 2 and 3 of Table 2. Both solutions have a coefficient of determination higher than 0.95 , illustrating the complexity in the analysis of ellipsometry results to evaluate correctly the film optical constants.

To elucidate the problem, in the second approach, the dispersion curve from the polarimetric method was used as an initial estimate in the ellipsometer analysis-modeling software, which then converges to a unique solution with $R^{2}=0.992$ (see column 5 in Table 2). This agrees well with the dispersion curve obtained in the first approach (column 2). Furthermore both approaches provide identical film thicknesses of $0.1954 \pm 0.0003 \mu \mathrm{m}$.

Other possibilities of applicability, as well as its limits, are discussed in the following section.

\section{Conclusion}

A characterization method was presented that combines spectral polarimetry and ellipsometry to uniquely provide the optical constants of dielectric thin films. The method uses polarimetry results as an initial estimate of the film dispersion curve. This result is independent of film thickness and is used to fit the ellipsometry fundamental equation to the experimental data obtained by spectral ellipsometry, preventing multiple and unrealistic solutions.

The validity of the proposed method was corroborated with a 42 -nm-thick $\mathrm{TiO}_{2}$ film for which ellipso- 
metry provides a unique solution at each spectral position.

For a thicker film with $195 \mathrm{~nm}$, for which ellipsometry provided two solutions, the spectral polarimetry estimate directed the route to the correct solution, illustrating the simplicity and usefulness of the combined method. For films with thicker optical thicknesses that may become multiples of halfwaves that cause destructive interference in reflection and therefore also produce reflectance matching, as mentioned in Section 2, these so-called absentee layer situations can be distinguished from the searched Brewster condition by simply noting that the former also occur with $s$-polarized light, unlike the latter.

Another important advantage of the combined method is that both techniques involved use the same experimental instrumentation, and measurements are performed in the same sample position, thus increasing reliability of results.

On the other hand, since the used polarimetric measurements are mostly sensitive to the neighborhood of the air-film interface [10], a large departure of the final ellipsometric dispersion data from the initial polarimetric values might occur. This is a limitation of the proposed method, which then can be used as an indicator of significant film inhomogeneity. Other limitations are intrinsic to ellipsometry itself, such as samples with too much scattering or requiring sophisticated models (anisotropic films for example) [14].

Finally, the presented method can be also extended to multilayer films. Similarly to ellipsometry, measurements can be performed in sequence, after each layer is individually deposited.

The authors are grateful for support from Conselho Nacional de Desenvolvimento Científico e Tecnológico (CNPq), Brazil, as well as to Laurent Kitzinger from Semilab USA LLC for his assistance with the control software of the SOPRA spectral ellipsometer photometric mode.

\section{References}

1. K. H. Zaininger and A. G. Revesz, "Ellipsometry: A valuable tool in surface research," RCA Rev. 25, 85-115 (1964).

2. M. Losurdo, M. Bergmair, G. Bruno, D. Cattelan, C. Cobet, A. de Martino, K. Fleischer, Z. Dohcevic-Mitrovic, N. Esser, M. Galliet, R. Gajic, D. Hemzal, K. Hingerl, J. Humlicek, R. Ossikovski, Z. V. Popovic, and O. Saxl, "Spectroscopic ellipsometry and polarimetry for materials and systems analysis at the nanometer scale: State-of-the-art, potential, and perspectives," J. Nanopart. Res. 11, 1521-1554 (2009).

3. R. Swanepoel, "Determination of the thickness and optical constants of amorphous silicon," J. Phys. E 16, 1214-1222 (1983).

4. P. K. Tien and R. Ulrich, "Theory of prism-film coupler and thin-film light guides," J. Opt. Soc. Am. 60, 1325-1337 (1970).

5. R. M. A. Azzam and N. M. Bashara, Ellipsometry and Polarized Light (North Holland 1987), Chaps. 3, 5, and 6.

6. H. G. Tompkins, A User's Guide to Ellipsometry (Academic 1993), Chap. 2.

7. F. Hiroyuki, Spectroscopic Ellipsometry: Principles and Applications (Wiley, 2007), Chap. 4.

8. F. Abelès, "Recherches sur la propagation des ondes électromagnétiques sinusoidales dans les milieux stratifiés. Application aux couches minces," Ph.D. thesis (Université de Paris, 1949) (in French).

9. M. Born and E. Wolf, Principles of Optics: Electromagnetic Theory of Propagation, Interference and Diffraction of Light (Cambridge Univ. Press 1997), Chap. 1.

10. F. Horowitz and M. B. Pereira, "Optical surface analysis of graded index coatings on glass," J. Non-Cryst. Solids 218, 286-290 (1997).

11. D. Brewster, "On the laws which regulate the polarisation of light by reflexion from transparent bodies," Phil. Trans. R. Soc. London 105, 125 (1815).

12. M. J. Hacskaylo, "Determination of the refractive index of thin dielectric films," J. Opt. Soc. Am. 54, 198-203 (1964).

13. P. A. Soave, R. A. F. Dau, M. R. Becker, M. B. Pereira, and F. Horowitz, "Refractive index control in bicomponent polymer films for integrated thermo-optical applications," Opt. Eng. 48, 1246031 (2009).

14. F. Horowitz, "Structure-induced optical anisotropy in thin films," Ph.D. thesis (Optical Sciences Center, University of Arizona, 1983), Tucson, EUA. Published by University Microfilms Inc. 